\title{
LOCATION OF THE CORE AND CONAL EMISSION REGIONS IN PSR 0329+54
}

\author{
J. A. GIL \\ The Astronomical Centre of Zielona Góra and Institute of Physics, Pedagogical University \\ M. V. Popov \\ Astro-Space Center of the Lebedev Physical Institute, Academy of Sciences
}

\begin{abstract}
We discuss the cross-correlation analysis of single pulses in the emission of PSR $0329+54$ observed simultaneously at 102.5 and $1700 \mathrm{MHz}$. We have found that the observed correlations between the two frequencies are consistent with the model in which the subpulses in single pulses correspond to plasma excitations flowing from the neutron-star surface along dipolar magnetic field lines. The peaks of strongly correlated subpulses at the two frequencies correspond to the same field line at two different emission radii. We have found that the core (central) and the conal (outer) components of the pulse profile of PSR $0329+54$ originate at the same distance from the neutron star.
\end{abstract}

\section{Introduction}

PSR $0329+54$ is probably one of the best examples of a pulsar with both core (central) and conal (outer) components (Rankin 1983a). The subpulses in single pulses occur at different longitudes across the profile but most frequently under the profile components. Kardashev et al. (1986) observed single pulses of this pulsar simultaneously at 102.5 and $1700 \mathrm{MHz}$. They found significant correlations between the two frequencies at some longitude intervals. Strong subpulses at $102.5 \mathrm{MHz}$ were highly correlated with strong subpulses at $1700 \mathrm{MHz}$. The times of arrival (TOA) of correlated subpulses at the two frequencies were associated according to a linear relationship (cf. figure 5 in Kardashev et al. 1986)

$$
t\left(\nu_{1}\right)=A t\left(\nu_{2}\right)+B
$$

where $\nu_{1}=1700 \mathrm{MHz}$ and $\nu_{2}=102.5 \mathrm{MHz}, A \approx$ 0.77 , and $B \approx 30.5 \mathrm{~ms}$. This relationship was exactly the same for both core (central) and conal (outer) components. We will discuss this important observational fact in the light of the standard magnetic-pole model of pulsar emission. We reproduce figure 5 of Kardashev et al. (1986) in our figure 1 .

\section{A standard model}

Let us consider a standard magnetic-pole model of pulsar emission. The relativistic particles emit radiation which is beamed tangent to the dipolar magnetic field lines. The emission is narrowband and the lower frequencies are emitted farther from the star than the higher ones. The subpulses correspond to plasma excitations which flow from the

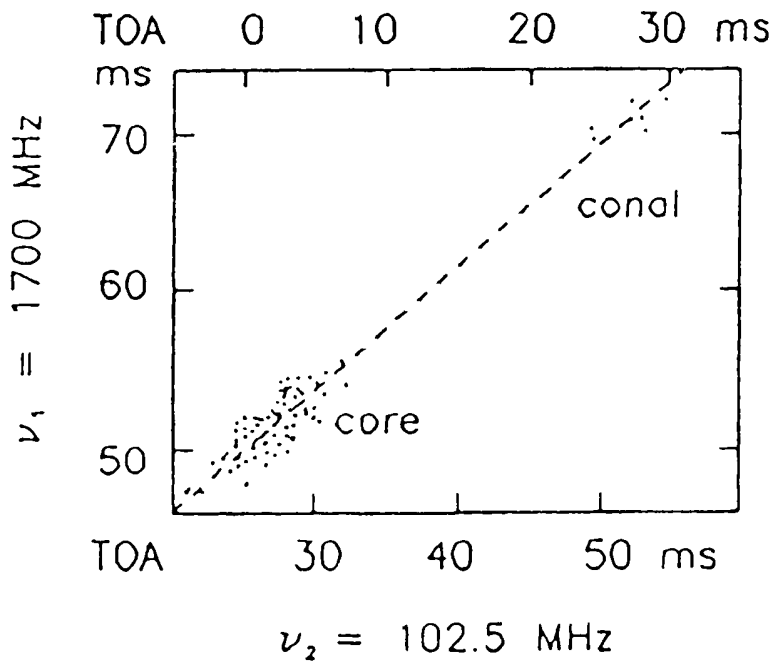

Figure 1 Correlations between the TOAs of strong subpulses at 102.5 and $1700 \mathrm{MHz}$ of PSR $0329+54$ (after Kardashev et al. 1986). The TOAs are corrected for dispersion time delays by profile alignment according to the known value of $D M$ (to within an accuracy of about $0.1 \%$ ). The two separate sets of data points correspond to the core (central) and conal (outer) components. The upper time axis for $102.5 \mathrm{MHz}$ is scaled in such a way that $t_{i}\left(\nu_{2}\right)=0$ corresponds to the center of the core component (profile midpoint). The dashed line describes a linear correlation between the two frequencies $t_{i}\left(\nu_{1}\right)=0.77 t_{i}\left(\nu_{2}\right)+11.25 \mathrm{~ms}$. This relationship is exactly the same for both the core and conal components.

stellar surface along the magnetic field lines. Therefore the peaks of strongly correlated subpulses observed at frequencies $\nu_{1}$ and $\nu_{2}<\nu_{1}$ correspond to the same field line at two different emission radii $r\left(\nu_{1}\right)$ and $r\left(\nu_{2}\right)>r\left(\nu_{1}\right)$. The subpulses observed at a single frequency at different pulse longitudes correspond to different field lines which will be labelled by the parameter $s_{i}=d_{i} / r_{\mathrm{p}}$, where $d_{i}$ is the distance from the magnetic axis to the base of the $i$-th field line at the polar cap surface, and $r_{p}$ is the 


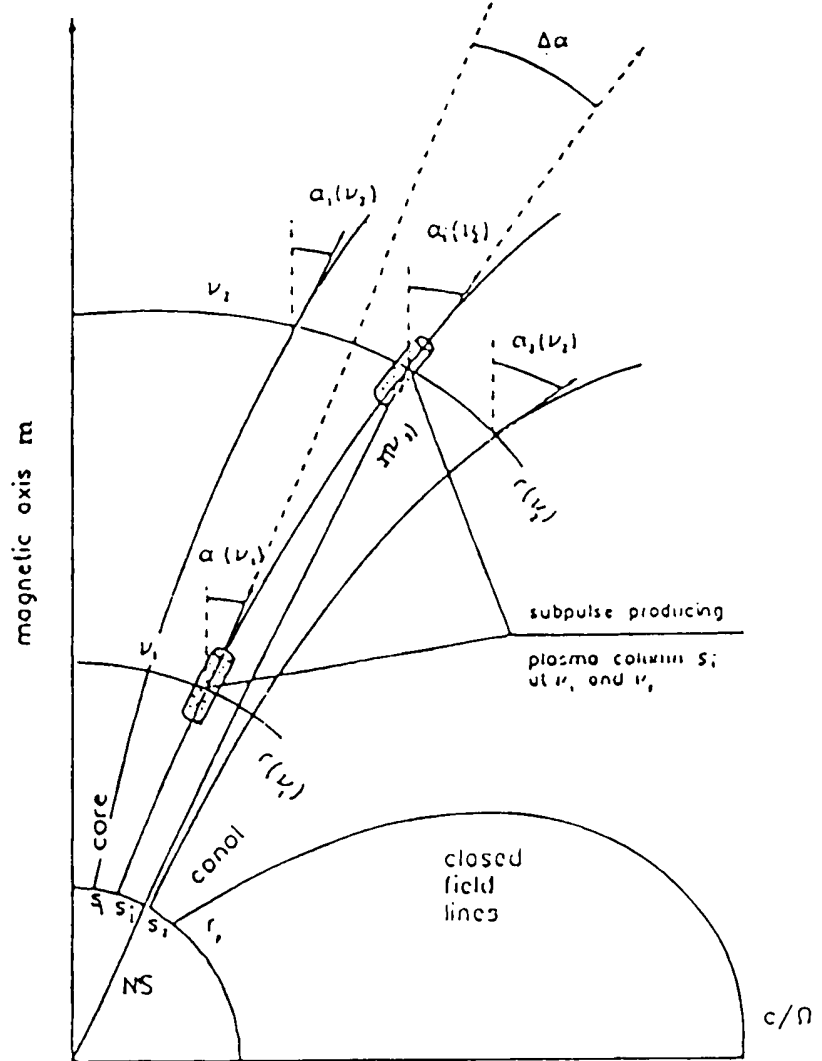

Figure 2 Geometry of the emission regions for PSR $0329+54_{4}$. At a given frequency an observer sees different field lines as the pulsar rotates. This means that the pulse profile of this pulsar represents a central cut through the polar cap, with core emission originating near the magnetic axis and conal emission originating near the polar cap edge. Therefore, we assume that the angle between the rotation and the magnetic axes and the angle between the rotation axis and observer's direction are almost the same in PSR 0329+54.

Goldreich-Julian (1969) polar cap radius (figure 2). In what follows we will assume that in PSR 0329+54 the pulse profile represents the central cut of the sight line through the polar cap, i.e. an observer passes very near the magnetic axis at the fiducial phase (profile center). This assumption is well justified by the presence of a strong core component in the pulse profile of this pulsar (see also Lyne and Manchester 1988).

The above model is illustrated in figure 2 . Let us consider the $i$-th subpulse associated with the $i$-th field line originating at a distance $d_{i}=s_{i} r_{\mathrm{p}}$ from the magnetic axis. The opening angle between the magnetic axis and the tangent to this line at different radii $r(\nu)$ is

$$
\alpha_{i}(\nu)=\frac{3}{2} s_{i}\left(\frac{\Omega}{c}\right)^{1 / 2} r^{1 / 2}(\nu),
$$

where $\Omega=2 \pi / P$ is the pulsar angular frequency, $P$ is the pulsar period, and $c$ is the speed of light. Let $t_{i}\left(\nu_{2}\right)$ be a TOA at frequency $\nu_{2}$ of the subpulse associated with $i$-th field line. In general, the corresponding TOA of the subpulse associated with the same field line at frequency $\nu_{1}$ can be expressed by the TOA at frequency $\nu_{2}$

$$
t_{i}\left(\nu_{1}\right)=t_{i}\left(\nu_{2}\right)-\Delta t_{\alpha}+\Delta t_{r}-\Delta t_{\mathrm{DM}}
$$

where

$$
\Delta t_{\alpha}=\frac{\Delta \alpha P}{2 \pi \sin \theta}+t_{0}
$$

$t_{0}$ is the arbitrary time corresponding to the fiducial phase in which $s=0$ and $\alpha\left(\nu_{2}\right)=\alpha\left(\nu_{1}\right)=0, \Delta t_{\alpha}$ is a time difference corresponding to different opening angles $\alpha_{i}\left(\nu_{1}\right)$ and $\alpha_{i}\left(\nu_{2}\right)$ as pulsar rotates, $\Delta \alpha=$ $\alpha_{i}\left(\nu_{2}\right)-\alpha_{i}\left(\nu_{1}\right)$ (figure 2), and $\theta$ is the angle between the rotation and magnetic axes.

$$
\Delta t_{r}=\left(\frac{1+\sin \theta}{c}\right)\left[r\left(\nu_{2}\right)-r\left(\nu_{1}\right)\right]
$$

is the retardation-aberration time delay between the two emission radii, and

$$
\Delta t_{\mathrm{DM}}=4.15 \times 10^{9} D M\left(\nu_{2}^{-2}-\nu_{1}^{-2}\right) \mu \mathrm{s}
$$

is the dispersion time delay between the two frequencies, where $D M$ is the dispersion measure.

According to eq.(2), the angular difference in eq.(4) for a dipolar magnetic field has the form

$$
\Delta \alpha_{i}=\frac{3}{2} s_{i}\left(\frac{2 \pi}{c P}\right)^{1 / 2}\left[r^{1 / 2}\left(\nu_{2}\right)-r^{1 / 2}\left(\nu_{1}\right)\right]
$$

and, after correcting for interstellar dispersion by profile alignment, eq. (3) can be written in the form

$$
\begin{aligned}
t_{i}\left(\nu_{1}\right)= & t_{i}\left(\nu_{2}\right)- \\
& \frac{(9 P / 8 \pi c)^{1 / 2} s_{i}}{\sin \theta}\left[r^{1 / 2}\left(\nu_{2}\right)-r^{1 / 2}\left(\nu_{1}\right)\right]+ \\
& t_{0}+\left(\frac{1+\sin \theta}{c}\right)\left[r\left(\nu_{2}\right)-r\left(\nu_{1}\right)\right]
\end{aligned}
$$

If one scales the $t\left(\nu_{2}\right)$ time axis in such a way that $t_{0}=0$, which means that $t_{i}\left(\nu_{2}\right)=0$ at the fiducial phase in which $\alpha\left(\nu_{2}\right)=0$ then

$$
t_{i}\left(\nu_{2}\right)=(9 P / 3 \pi c)^{1 / 2} s_{i} r^{1 / 2}\left(\nu_{2}\right) / \sin \theta
$$

and

$$
\begin{aligned}
t_{i}\left(\nu_{1}\right)= & t_{i}\left(\nu_{2}\right)\left[\frac{r\left(\nu_{1}\right)}{r\left(\nu_{2}\right)}\right]^{1 / 2}+ \\
& \left(\frac{1+\sin \theta}{c}\right)\left[r\left(\nu_{2}\right)-r\left(\nu_{1}\right)\right],
\end{aligned}
$$

which explains the linear relationship between the TOAs at 102.5 and $1700 \mathrm{MHz}$ presented in figure 1 (corrested for interstellar dispersion by profile alignment according to the known value of $D M$ ) and expressed in eq.(1). Moreover, as can be seen from 
figure 1 the linear relationship between TOAs at $102.5 \mathrm{MHz}$ and $1700 \mathrm{MHz}$ in PSR $0329+54$ is the same for core and conal subpulses. This means that the coefficients $A$ and $B$ in eq.(1) are the same for both the core and conal components. According to eq. (8) this, in turn, means that

$$
\left.\frac{r\left(\nu_{1}\right)}{r\left(\nu_{2}\right)}\right|_{\text {core }}=\left.\frac{r\left(\nu_{1}\right)}{r\left(\nu_{2}\right)}\right|_{\text {cone }}
$$

and

$$
r\left(\nu_{2}\right)_{\text {core }}-r\left(\nu_{1}\right)_{\text {core }}=r\left(\nu_{2}\right)_{\text {cone }}-r\left(\nu_{1}\right)_{\text {cone }} .
$$

The above conditions have a very important interpretation: core and conal emission originate at the same altitude $r(\nu)_{\text {core }}=r(\nu)_{\text {cone }}$ in PSR $0329+54$. The core emission originates closer to the magnetic axis and the conal emission closer to the polar cap edge, i.e., $s_{\text {core }}<s_{\text {cone }} \approx 1$, $\left[t_{i}(\nu) \propto \alpha_{i}(\nu) \propto s_{i} r^{1 / 2}(\nu)\right]$.

\section{Conclusions}

The important conclusion of this paper is that the emission associated with the central component (core) and outer components (conal) of the mean profile of PSR $0329+54$ originates at the same distance from the neutron star. This is in strong contradiction to Rankin (1990) who argues that the core emission originates essentially at the neutronstar surface and occupies the entire polar cap. Within this interpretation conal components would originate further from the polar cap, in contradiction to results of this paper. 